\title{
Ab 2018: Höherer Mindestlohn in der Pflege
}

\section{Gute Nachricht vor allem für Pflegehilfskräfte}

॥ Die Empfehlung der Pflegekommission war einstimmig: $\mathrm{Ab}$ 1. Januar 2018 soll der Mindestlohn in der Pflege

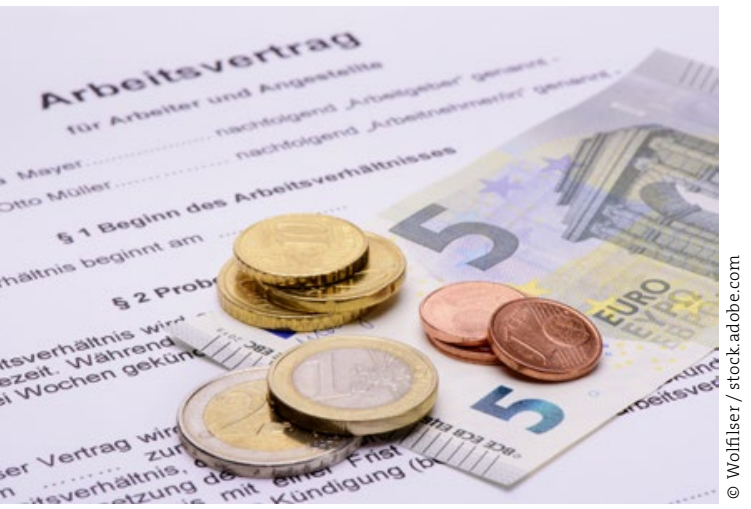

auf 10,55 Euro pro Stunde im Westen und 10,05 Euro im Osten steigen. Bis 2020 soll er in zwei Schritten weiter angehoben werden, und zwar auf 11,35 Euro pro Stunde im Westen und 10,85 Euro im Osten. Davon profitieren gerade Pflegehilfskräfte. Bundesarbeitsministerin Andrea Nahles kommentierte die Empfehlung so: „Der Bedarf an guter, qualifizierter und motivierter Arbeit wächst in allen Pflegebereichen. Der Mindestlohn ist ein Beitrag dazu, die Qualität in diesem Berufsfeld zu sichern und faire Wettbewerbsbedingungen für die Einrichtungen zu schaffen." Und Rainer Brückers, Beauftragter des BMAS für die Pflegekommission, sieht es als ,ein wichtiges Signal für die Branche, dass die Pflegekommission wiederum ein einvernehmliches Ergebnis zur Anpassung der Pflegemindestlöhne erzielt hat."

Rund 900.000 Beschäftigte arbeiten aktuell in Einrichtungen, die unter den Pflegemindestlohn fallen. Wo der spezielle Pflegemindestlohn nicht gilt, z.B. in Privathaushalten, gilt der allgemeine gesetzliche Mindestlohn. Das Bundesministerium für Arbeit und Soziales strebt an, auf Grundlage der Empfehlung der Pflegekommission den neuen Pflegemindestlohn auf dem Weg einer Verordnung zu erlassen.

www.bmas.de

\section{Deutschen Preis für Patientensicherheit 2017}

\section{Initiative für mehr Rezeptqualität: Universitätsklinikum Heidelberg erhält ersten Platz}

॥ Ärzte führen Tausende von Gesprächen - und müssen ihren Patienten auch schlechte Nachrichten überbringen. Gerade dann ist eine intensive Kommunikation wichtig, denn schnell können Ängste entstehen. Studien zeigen, dass gute Arzt-Patienten-Gespräche zu besseren Behandlungsergebnissen führen. Demzufolge gefährdet eine unzureichende Kommunikation die Patientensicherheit.

Nicht nur Arzt-Patienten-Gespräche, sondern auch der Austausch zwischen Ärzten und Pflegenden spielt im Gesundheitswesen eine wichtige Rolle. „Alle Mitarbeiter in Gesundheitseinrichtungen - und insbesondere diejenigen auf der Führungsebene - tragen die Verantwortung für eine gute Sicherheitskultur in der Patientenversorgung“, sagt Hedwig François-Kettner auf der 12. Jahrestagung des Aktionsbündnisses Patientensicherheit e.V. (APS). „Dazu gehört auch eine Atmosphäre, in der angstfrei gesprochen werden kann", so die Vorsitzende des APS. Es sei wichtig, in jeder Hierarchie kritische Themen und Schwachstellen benennen zu können.
Über 100 Referenten gaben auf der Tagung Anregungen und Erfahrungswerte für eine gelungene Kommunikation im Gesundheitswesen. Neben Vorträgen standen praktische Übungen auf dem Programm: Mehr als 20 Workshops behandelten unterschiedliche Aspekte der - auch digitalen - Kommunikation im Gesundheitswesen. So zeigen Referenten etwa, wie Schüler und Studenten eine gute Kommunikation schon in der Ausbildung und im Studium lernen und üben können.

Ein Höhepunkt der Veranstaltung war die Verleihung des Deutschen Preises für Patientensicherheit. Aus über 40 eingereichten Projekten sind besonders nachhaltige Best-PracticeBeispiele ausgewählt worden. Sieger wurde eine Initiative zur Erhöhung der Rezeptqualität des Universitätsklinikums Heidelberg.

(how)

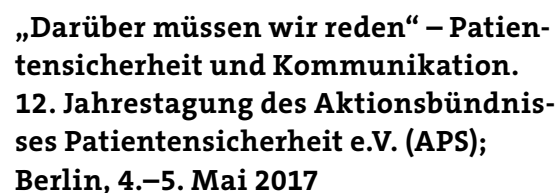

„Darüber müssen wir reden“ - Patientensicherheit und Kommunikation. 12. Jahrestagung des AktionsbündnisBerlin, 4.-5. Mai 2017

\section{Klares Bekenntnis}

\section{DBfK bezieht Stellung}

॥ Der Deutsche Berufsverband für Pflegeberufe (DBfK) hat aus gegebenem Anlass ein Positionspapier gegen Diskriminierung, Rassismus und Extremismus veröffentlicht. „Mit großer Sorge nehmen wir wahr, dass extremes Gedankengut, Aggression und Gewalt in unserer Gesellschaft zunehmend Fuß fassen. Opfer sind vor allem vulnerable Personen und Gruppen, die in besonderem Maße unseren Schutz brauchen. Populisten greifen Unzufriedenheit und Gefühle von Ungerechtigkeit in der Bevölkerung auf und nutzen sie für eigene Zwecke. Mit Hassparolen und Scheinlösungen wird versucht, das demokratische Wertesystem auszuhöhlen, das unserer Verfassung zugrunde liegt. Als professionell Pflegende können wir dazu nicht schweigen, sondern distanzieren uns ausdrücklich von solchem Gedankengut. In unserem Beruf haben wir uns dem ICN-Ethikkodex verpflichtet und stehen - über den Berufsalltag hinaus - für Toleranz und Solidarität", so DBfKPräsidentin Christel Bienstein. „Pflegerisches Handeln und Extremismus in jeglicher Form sind unvereinbar!“

www.dbfk.de 